\title{
Management of low rectal gastrointestinal stromal tumor with neoadjuvant therapy and transanal excision: a rare case report and review of the literature
}

This article was published in the following Dove Medical Press journal:

International Journal of General Medicine

\author{
Christopher Kosmidis ${ }^{1,2}$ \\ Konstantinos Sapalidis' \\ Alexandros Tsakalidis' \\ Stefanos Atmatzidis' \\ Nikolaos Michalopoulos' \\ Georgios Koimtzis' \\ Georgia Karayannopoulou ${ }^{3}$ \\ Sofia Lypiridou ${ }^{3}$ \\ Nikolaos Varsamis ${ }^{2}$ \\ Georgios Kouklakis² \\ Sofia Baka ${ }^{2}$ \\ Paul Zarogoulidis' \\ Isaac Kesisoglou' \\ 'Third Department of Surgery, \\ AHEPA University Hospital, \\ Aristotle University of Thessaloniki, \\ Thessaloniki, Greece; ${ }^{2}$ Department of \\ Oncology, Interbalkan Medical Center, \\ Thessaloniki, Greece; ${ }^{3}$ Department \\ of Pathology, Aristotle University of \\ Thessaloniki, Thessaloniki, Greece
}

\begin{abstract}
Background: Gastrointestinal stromal tumors (GISTs) are the most common mesenchymal tumors of the gastrointestinal tract. However, rectal GISTs represent only $5 \%$ of this category. We report a case of a rectal GIST treated with local excision after neoadjuvant therapy.

Case presentation: A 41-year-old male patient presented with anal bleeding. Colonoscopy revealed a mass located $5 \mathrm{~cm}$ from the anal verge. Histological examination showed a GIST with immunohistochemical positivity for CD117 and CD34. Transanal local excision was performed after neoadjuvant therapy.
\end{abstract}

Conclusion: Neoadjuvant immunotherapy for GISTs with unfavorable localization may facilitate local excision and avoid complications of more demanding operations.

Keywords: gastrointestinal stromal tumor, rectal, neoadjuvant therapy, transanal excision

\section{Background}

Gastrointestinal stromal tumors (GISTs) are the most common mesenchymal tumors of the gastrointestinal tract. GISTs are typically found in older adults $>50$ years old, and their main location is the stomach $(60 \%)$ followed by the small bowel $(30 \%)$, duodenum $(5 \%)$, colon and rectum $(<5 \%)$, esophagus and appendix $(<1 \%){ }^{1}$

Complete excision is the main treatment. Due to the known mutation in the expression of CD117 protein in GISTs, tyrosine kinase inhibitors such as imatinib or the newest sunitinib can be helpful for the treatment of primary unresectable tumors and metastatic disease or as an adjuvant therapy to reduce the rate of recurrence. ${ }^{2}$

We present a case of a rectal GIST in a young male patient, who was treated with neoadjuvant therapy and local excision.

\section{Case presentation}

A 41-year-old male patient presented to our department with the symptom of anal bleeding. The digital rectum examination revealed a palpable mass. Colonoscopy was performed, which showed a well-defined mass located on the left-anterior aspect of the anal canal at $\sim 5 \mathrm{~cm}$ from the anal verge and biopsies were taken. Abdominal computed tomography (CT) and MRI scan confirmed the presence of an intraluminal mass with a diameter of $5.5 \mathrm{~cm}$. The histological examination of the biopsy revealed a GIST immunohistochemically positive for CD117 and CD34.

Due to the size of the tumor, the decision to treat the patient with neoadjuvant therapy with imatinib $400 \mathrm{mg}$ once a day for 3 months was taken, in order to avoid
Correspondence: Paul Zarogoulidis Third Department of Surgery, AHEPA University Hospital, Aristotle University of Thessaloniki, 64 Panorama ST,

Thessaloniki, 55236, Greece

Tel +30697727 I974

Email pzarog@hotmail.com 
colectomy and facilitate local resection of the tumor. The posttreatment MRI demonstrated a notable reduction in tumor size $(2 \times 2 \times 1.8 \mathrm{~cm})$. Based on this finding, the patient was scheduled for surgery, and a transanal local excision of the tumor was performed (Figure 1).

Gross pathological examination of the specimen revealed a $4 \times 2 \times 1.8 \mathrm{~cm}$ mass (Figure 2 ), in which a tan-white $1.9 \mathrm{~cm}$ lesion was recognized on sectioning. Histological examination of this lesion showed a tumor composed of spindle or polygonal cells, with eosinophilic cytoplasm, poorly defined cell membranes, and round or elongated nuclei forming palisades with occasional perinuclear vacuolization. The neoplastic cells were arranged in intersecting bundles and occasionally in whorls. No necrosis was observed. The mitotic rate was mild $(<5$ mitoses per 50 high-power fields), and there was no evidence of tumor cells in surgical margins (R0 excision; Figures 3 and

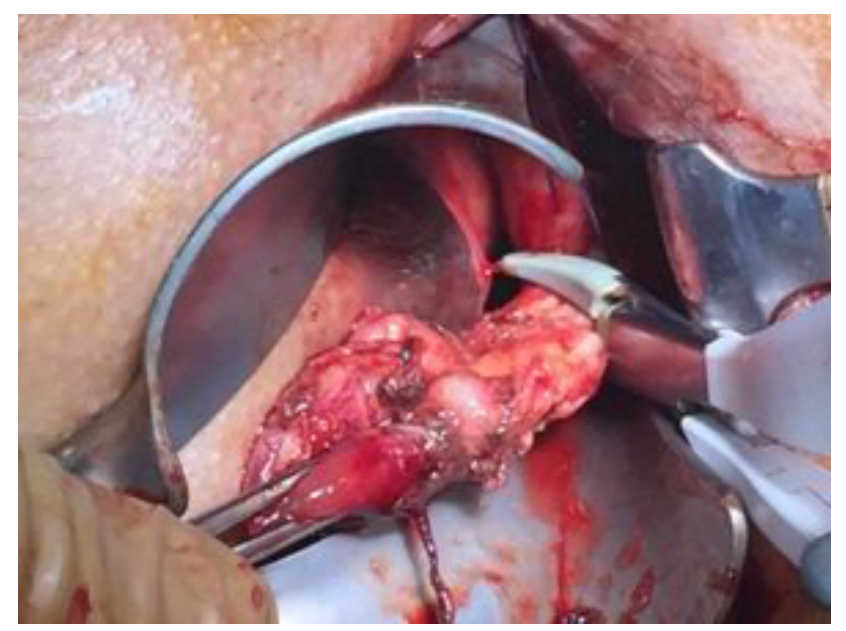

Figure I Transanal local excision of the tumor.

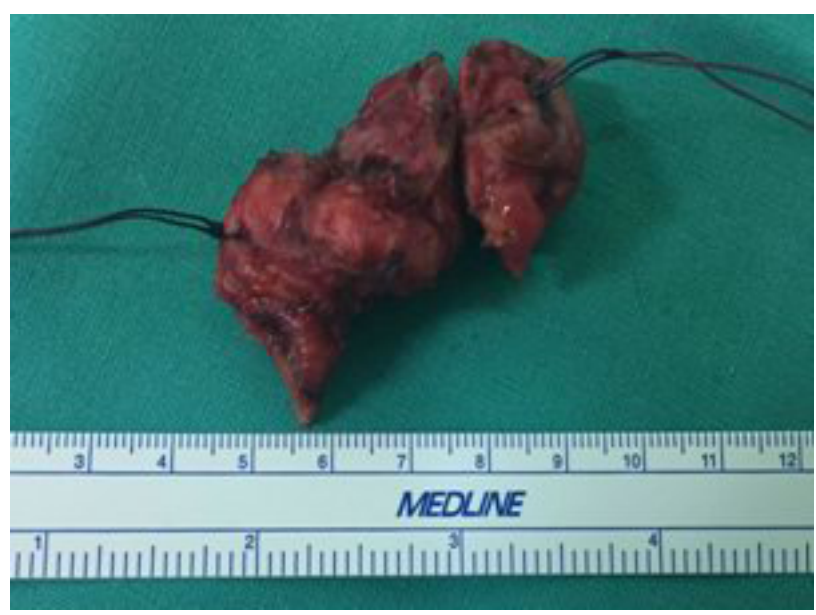

Figure 2 Rectal gastrointestinal stromal tumors specimen.
4). Immunohistochemically, neoplastic cells were diffuse and strong stained for C-KIT (CD117) and CD34 but not for smooth muscle actin (Figures 5-7).

The postoperative course was uneventful and the patient was discharged on the first postoperative day. Adjuvant therapy with imatinib resumed and a routine follow-up with a CT scan and colonoscopy was scheduled every 6 months. At present, 18 months postoperatively, the patient remains free of disease.

\section{Discussion}

GISTs represent almost $80 \%$ of the mesenchymal neoplasms of the gastrointestinal tract. The neoplastic GIST cells originate from a common precursor cell, which gives rise to the interstitial cells of Cajal. These cells are located in the

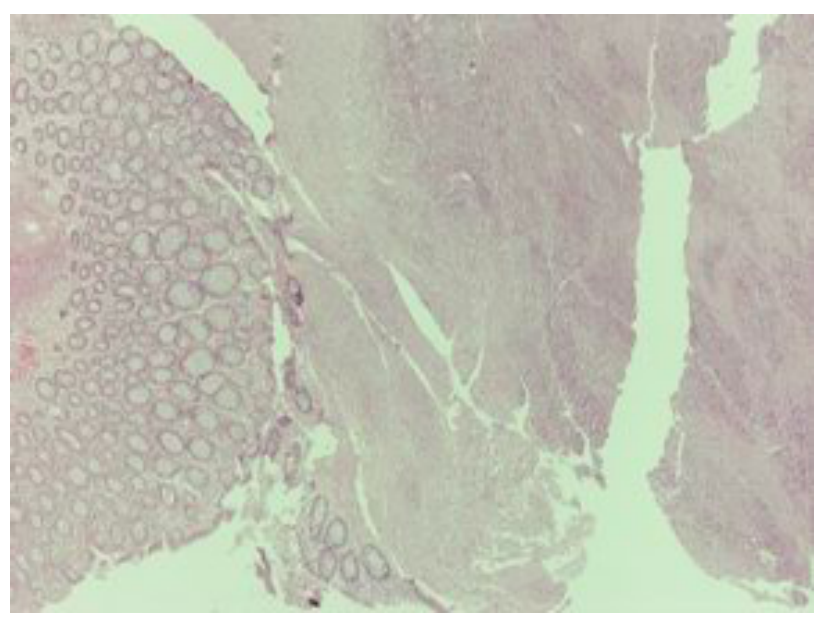

Figure 3 Microscopic features, $\mathrm{H} \& \mathrm{E} \times 10$.

Notes: Tumor composed of spindle or polygonal cells with eosinophilic cytoplasm, parts of which are arranged like woven, vortex or in intersecting bundles.

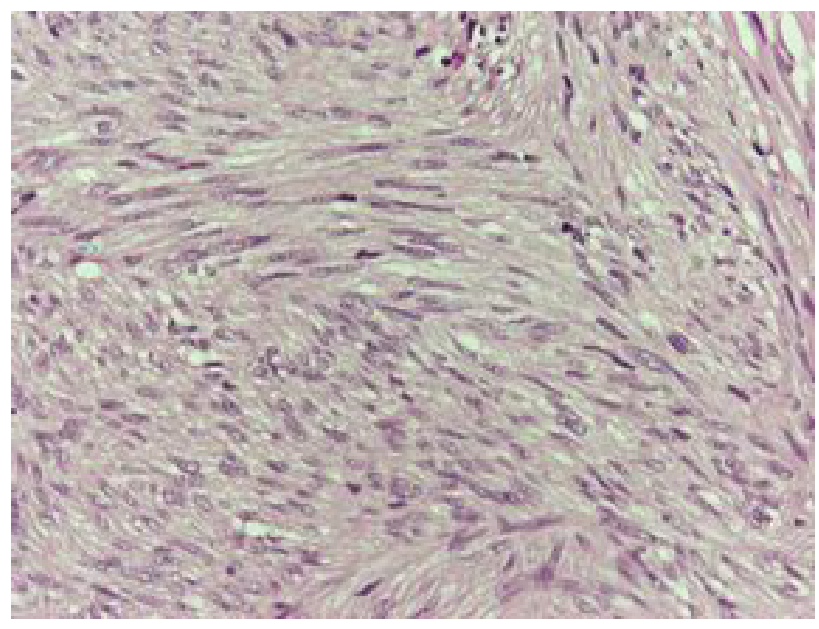

Figure 4 Microscopic features, $\mathrm{H} \& \mathrm{E} \times 40$.

Notes: Tumor composed of spindle or polygonal cells with eosinophilic cytoplasm, parts of which are arranged like woven, vortex or in intersecting bundles. 


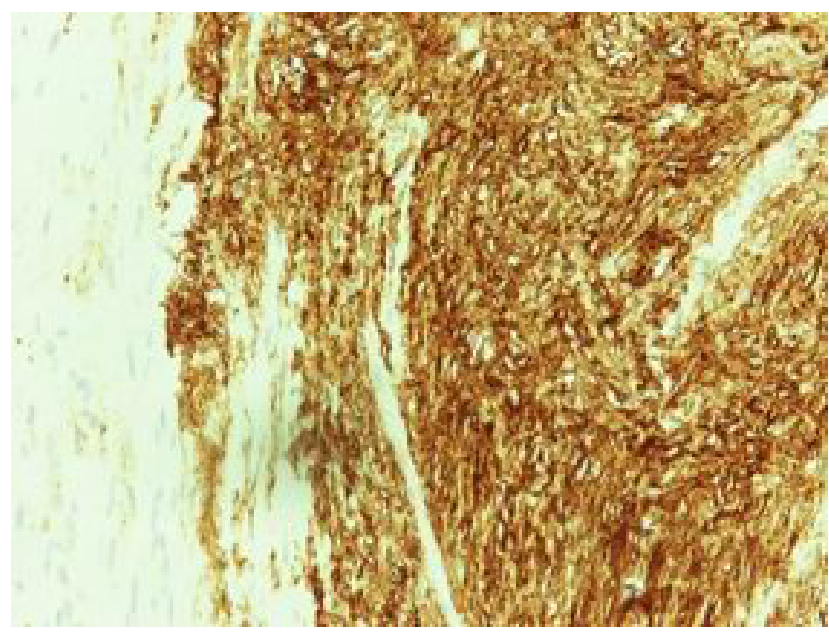

Figure 5 Immunohistochemical stain positive for C-KIT $(\times 40)$.

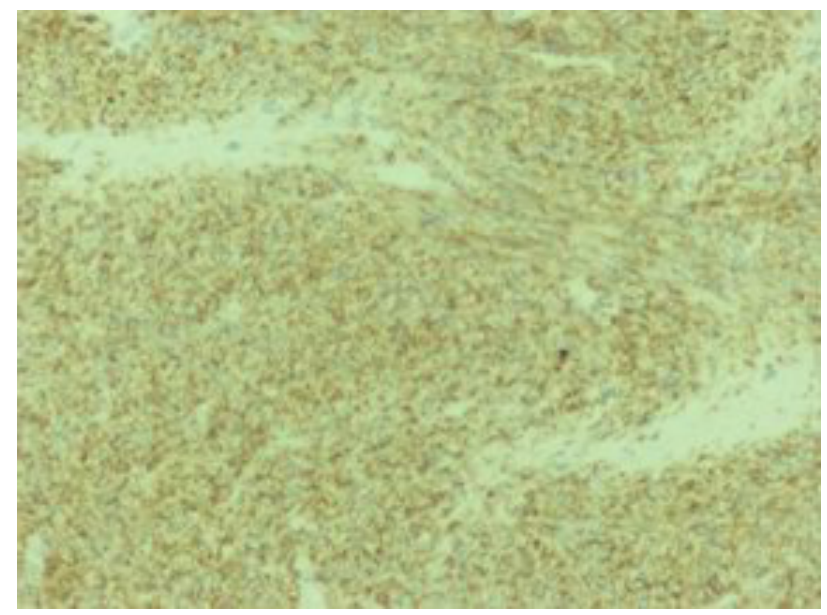

Figure 6 Immunohistochemical stain positive for CD34 $(\times 10)$.

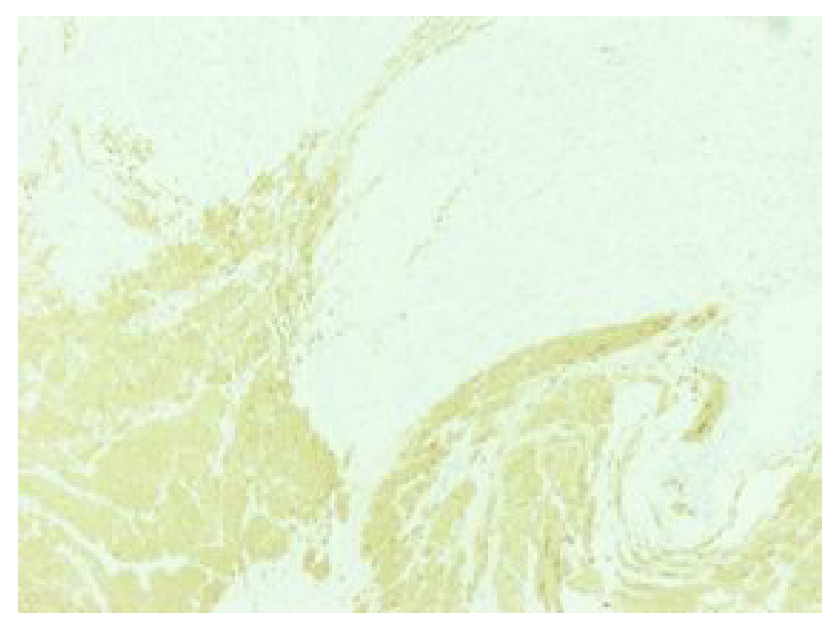

Figure 7 Immunohistochemical stain negative for smooth muscle actin $(\times 10)$. myenteric plexus of the gastrointestinal wall and serve as a pacemaker of the gut. ${ }^{1}$

GISTs are usually located in the stomach (60\%); the most common site of metastasis is the liver, whereas lymph node metastases are extremely rare. ${ }^{1}$ Recurrence can develop up to $10-15$ years after primary treatment, requiring therefore long-term clinical follow-up. ${ }^{2}$

GISTs are characterized from a mutation in the gene that is responsible for the expression of the CD117 (C-KIT) protein, a tyrosine kinase growth factor receptor. CD34 is also frequently expressed, but is less specific than CD117. ${ }^{3}$

The main prognostic factors of malignancy are mitotic activity, tumor size, and tumor origin. GISTs with high mitotic rate over 5 mitoses per 50 high-power fields (a total area of 5 $\mathrm{mm}^{2}$ ), bigger size, and localization other than usual are related to a higher risk of malignancy. ${ }^{4,5}$ Complete surgical excision remains the gold standard for the treatment of GISTs. ${ }^{1,6}$ The less extensive excision that achieves the R0 resection is the preferable method. ${ }^{7}$ Therefore, local transanal excision is the treatment of choice for lesions situated in mid or low rectum. ${ }^{8,9}$

GISTs fail to respond to conventional chemotherapy and radiation; ${ }^{10}$ thus, adjuvant therapy with a tyrosine kinase inhibitor such as imatinib is strongly recommended in highrisk patients to reduce the risk of recurrence. ${ }^{11}$ Adjuvant therapy should not be applied when the risk of aggressive behavior is low. ${ }^{4}$

Neoadjuvant therapy (oral imatinib $400 \mathrm{mg}$ for 4-6 months $\mathbf{s}^{12,13}$ ) is applied when the tumor is primary unresectable or due to its localization a more extensive surgical procedure is required. Oral treatment is generally well tolerated, and the majority of patients achieve complete or partial remission $^{5}$ and reduction of the mitotic rate. ${ }^{3}$ However, mutational analysis is crucial to distinguish the resistant genotypes that will not respond to therapy, in order to avoid any delay of the surgical excision. ${ }^{4}$

Low rectal GISTs are relatively rare and are usually related to more aggressive behavior than gastric GISTs. ${ }^{1}$ It is an interesting subgroup of the GISTs, because of their large size and the proximity to the anal sphincter. Due to their location, abdominoperineal resection, with possible complications such as sexual or urological dysfunctions, ${ }^{14}$ is sometimes unavoidable. Therefore, neoadjuvant treatment with tyrosine kinase inhibitors may be necessary in order to achieve local complete resection with anus preservation, as in the case we present. Imatinib can be safely discontinued 2-3 days before surgery and when the patient recovers from the operation can be resumed immediately. ${ }^{4}$ 


\section{Conclusion}

Low rectal GISTs are a rare entity that requires multidisciplinary management and long-term surveillance. Neoadjuvant therapy with imatinib and downstaging of the tumor plays an important role in order to facilitate a successful local R0 excision and avoid more extensive surgical procedures related to a higher risk of complications.

\section{Ethics statement}

Written informed consent has been provided by the patient to have the case details and any accompanying images published. Investigational review board approval was acquired for the publication of the case report from the Investigational Review Board of the Third Surgery Department of AHEPA University Hospital, Aristotle University of Thessaloniki, Thessaloniki, Greece, although it was not required.

\section{Disclosure}

The authors report no conflicts of interest in this work.

\section{References}

1. Demetri GD, von Mehren M, Antonescu CR, et al. NCCN Task Force report: update on the management of patients with gastrointestinal stromal tumors. J Natl Compr Canc Netw. 2010;8(Suppl 2): S1-S41.

2. Miettinen M, Lasota J. Gastrointestinal stromal tumors: review on morphology, molecular pathology, prognosis, and differential diagnosis. Arch Pathol Lab Med. 2006;130(10):1466-1478.
3. Jiang Z-X, Zhang SJ, Peng WJ, Yu BH. Rectal gastrointestinal stromal tumors: imaging features with clinical and pathological correlation. World J Gastroenterol. 2013;19(20):3108-3116.

4. ESMO/European Sarcoma Network Working Group. Gastrointestinal stromal tumours: ESMO clinical practice guidelines for diagnosis, treatment and follow-up. Ann Oncol. 2014;25(Supp 3): iii21-iii26.

5. Miettinen M, Lasota J. Gastrointestinal stromal tumors-definition, clinical, histological, immunohistochemical, and molecular genetic features and differential diagnosis. Virchows Archiv. 2001;438(1):1-12.

6. Theodoropoulos D. Gastrointestinal tumors of the colon and rectum. Clin Colon Rectal Surg. 2011;24(03):161-170.

7. Nigri GR, Dente M, Valabrega S, et al. Gastrointestinal stromal tumor of the anal canal: an unusual presentation. World J Surg Oncol. 2007;5(1):20.

8. Pai VD, Demenezes JL, Patil PS, Saklani AP. Multimodality therapy of rectal gastrointestinal stromal tumors in the era of imatinib-an Indian series. J Gastrointest Oncol. 2016;7(2):262-268.

9. Centonze D, Pulvirenti E, Pulvirenti D'Urso A, Franco S, Cinardi N, Giannone G. Local excision with adjuvant imatinib therapy for anorectal gastrointestinal stromal tumors. Tech Coloproctol. 2013;17(5): 571-574.

10. Atmatzidis S, Chatzimavroudis G, Ananiadis A, Kapoulas S, Atmatzidis $\mathrm{K}$. Giant GIST of the small intestine in a young man. $J$ Gastrointest Surg. 2013;17(8):1536-1537.

11. Joensuu H, Eriksson M, Sundby Hall K, et al. One vs three years of adjuvant imatinib for operable gastrointestinal stromal tumor. JAMA. 2012;307(12):1265-1272.

12. Verweij J, Casali PG, Zalcberg J, et al. Progression-free survival in gastrointestinal stromal tumours with high-dose imatinib: randomised trial. Lancet. 2004;364(9440):1127-1134.

13. Machlenkin S, Pinsk I, Tulchinsky H, et al. The effect of neoadjuvant imatinib therapy on outcome and survival after rectal gastrointestinal stromal tumour. Colorectal Dis. 2011;13(10):1110-1115.

14. Fiore M, Raut CP, Gronchi A. Are we allowed to limit surgical aggressiveness with small rectal gastrointestinal stromal tumors? Ann Surg Oncol. 2017;24(5):1153-1156.
International Journal of General Medicine

\section{Publish your work in this journal}

The International Journal of General Medicine is an international, peer-reviewed open-access journal that focuses on general and internal medicine, pathogenesis, epidemiology, diagnosis, monitoring and treatment protocols. The journal is characterized by the rapid reporting of reviews, original research and clinical studies across all disease areas.

\section{Dovepress}

The manuscript management system is completely online and includes a very quick and fair peer-review system, which is all easy to use. Visit http://www.dovepress.com/testimonials.php to read real quotes from published authors. 